\title{
No Claim Bonus?
}

\author{
by John D. Hey*
}

\section{Introduction}

No claim bonus schemes are a prominent feature of some insurance contracts, most notably those for motor insurance. Given the existence of such a scheme, the policy-holder has to decide whether the magnitude of any accident is sufficiently great to justify a claim, since making a claim necessarily involves a future loss of discount. This decision-problem is not a trivial one, since the loss of discount extends over a number of future years.

The first purpose of this paper is the formulation of the appropriate decision rule. We adopt a dynamic infinite horizon specification which enables full incorporation of the dynamic implications of the outcome of the decision whether to claim. We also assume that the decision-maker is risk-averse, thus justifying the decision to purchase insurance in the first place. These specifications represent significant improvements on previous contributions to the literature (Lemaire [1976] and [1977], Venezia and Levy [1980] and Zweifel and Gnaedinger [1983]).

We then go on to examine the implications, for both the policy-holder and the insurance company, of the existence of a no claim bonus scheme. Specifically, we examine how the introduction of such a scheme alters: (a) the profits of the insurance company (given a specified level of utility for the policy-holder), and (b) the utility of the policy-holder (given a specified rate of profits for the company). Interestingly, we show that, for realistic orders of magnitudes for the various parameters of the problem, the introduction of a no claim bonus scheme has an almost insignificant effect. Thus the empirical justification for the continuing existence of such bonus schemes appears to be in some considerable doubt. This would appear to confirm expert opinion within the actuarial profession (see, for example, Bennett [1974]).

We discuss these latter issues in some detail in section 5. In the meantime, we formulate and solve the problem of deciding whether or not to claim. To be specific, in the next section we describe our theoretical framework and assumptions and introduce our notation. In section 3 we present the solution to the problem, and discuss its properties. Section 4 provides a number of specific numerical examples which shed light on the relevant orders of magnitudes

* Professor of Economics and Statistics, University of York. I am greatly indebted to my father, G. B. Hey, M.A., F.I.A., for many invaluable discussions and for much valuable inside information on this topic. Having a father who is an expert on motor insurance is a bonus I have claimed frequently; my thanks to him. (Like all good insurance companies, he will probably deny any liability for what follows.) 
and presents some comparative static results. Section 5, as we have already noted, then turns to examine the impact (on profits and utility) of the introduction of a no claim discount scheme. Finally, section 6 offers some comments in conclusion.

\section{Assumption and notation}

This section sets up the problem of the optimal claim strategy as viewed by the policy-holder. For expository purposes, we talk throughout in terms of motor insurance. But, of course, our model carries over to other kinds of insurance which also operate a no claim discount scheme.

We assume that the policy-holder lives either for an infinite number of periods (where the period coincides with the length of a typical insurance contract) or for random number of periods (with the date of death being determined by a memoryless stochastic device - see later). We assume that he or she receives a constant income $I$ per period while alive, which is spent either on car insurance and car accident repairs or on consumption. We denote the latter by $C$. We assume that only $C$ yields utility, and hence that expenditure on car insurance or car accident repairs does not yield utility. Further, we assume that lifetime utility as viewed from the beginning of period $T$ is given by

$$
\sum_{t=T}^{\infty} \rho^{t-T} U\left(C_{t}\right)
$$

where $\rho$ is the constant discount rate and $U(C)$ is the utility gained in some period from consuming an amount $C$ in that period. We assume that $U^{\prime}>0$, and, where necessary, that $U^{\prime \prime}<0$. In any period, $C_{t}$ is given by

$$
C_{t}=I-E_{t}
$$

where $E$ is the expenditure on car insurance and car accident repairs in period $t$. (We note that, for an individual with an infinite life, $\rho$ is the true discount rate, while for a random-life individual $\rho$ is the true discount rate multiplied by $\theta$, where $\theta$ is the assumed-constant probability that the individual is alive in period $t+1$ given that he or she was alive in period $t$.) Finally, we assume that the individual's objective is to maximize the expected value of lifetime utility (as given by (l)).

So much for the individual. Let us now specify the details of the insurance contract.

We assume that the insurance contract contains $N$ premium rates (or categories) $n=1, \ldots$, $N$. We denote the premiums in these categories by $P_{1}, P_{2}, \ldots, P_{N}$ respectively. We assume that $P_{1}>P_{2}>\ldots>P_{N}$. A premium payment provides insurance for one period. The individual's premium changes from period to period according to whether he or she makes a claim in a particular period, the precise mechanism depending upon the specific no claim discount scheme operated by the insurance company. Now, in real life, an individual may have more than one accident in any one period, and thus may face a claim-decision more than once within a period. To model this completely would complicate our model enormously without adding much in insight. So we will simplify: we assume that the individual can effectively wait until the end of the period before deciding whether to claim or not, and, moreover, can take this decision in the light of the total accident bill for the period in question. 
So let us denote by $p$ the probability that the individual has at least one accident in any given period, and let $F(x)$ denote the distribution function of the total accident bill $x$ in any given period (with $f(x)$ denoting the corresponding probability distribution function). We assume that $p, F($.$) , and hence f($.$) , are constant through time.$

So in any one period, the individual either claims off the insurance or does not. Let us suppose that the no claims discount scheme operated by the insurance company specifies that the premium category in a given period depends on two things: (1) the premium category in the immediately preceding period; and (2) whether or not a claim was made in that preceding period. (This is the normal procedure, through some companies take a longer period of history into account.) We can characterise any such scheme by a rule of the following form:

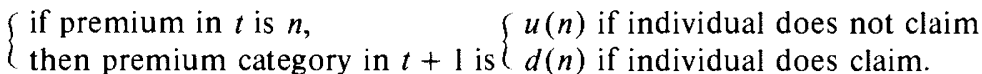

Thus, if the premium payment in $t$ is $P_{n}$ then, if the individual claims in period $t$, the premium in $t+1$ is $P_{d(n)}$; otherwise it is $P_{u(n)}$. We will assume that $n \leqslant u(n) \leqslant N$ and that $1 \leqslant d(n) \leqslant n$. (Recall that $P_{1}>P_{2}>\ldots>P_{N}$, so that $P_{1}$ is the highest premium and $P_{N}$ the lowest premium.) Normally, but not exclusively, an individual with no insurance-experience will start in category 1.

In practice, there is a variety of no claim discount schemes. A particularly simple scheme, which we term the "one up/one down scheme" is characterised by

$$
\begin{cases}u(n)=n+1,1 \leqslant n \leqslant N ; & d(1)=1 ; \\ u(N)=N . & d(n)=n-1,2 \leqslant n \leqslant N .\end{cases}
$$

This rewards individuals for each claim-free period by a reduction in premium to the next lowest category; each claim is "punished" by an increase in the premium to the next highest category. (There are obvious modifications at the extremes.) Most schemes have harsher "punishments" than in the "one up/one down scheme": fairly typical is an increase of 2 in the premium category after a claim. This is illustrated in what we term the "majority scheme", which is characterised by

$$
\begin{cases}u(n)=n+1,1 \leqslant n \leqslant N ; & d(n)=1,1 \leqslant n \leqslant 2 \\ u(N)=N . & d(n)=n-2,3 \leqslant n \leqslant N .\end{cases}
$$

Finally, we denote by $e_{n}$ the deductible paid when in category $n(n=1, \ldots, N)$ under the insurance contract; thus, if the individual is in category $n$ in a particular period, and if he or she makes a claim that period, then the first $e_{n}$ of the claim is paid by the individual.

To summarise: in any given period, the individual is in some category $n$ and pays premium $P_{n}$; with probability $p$ "an" accident occurs, the total accident cost being an independent drawing from the distribution $F($.$) ; if no accident occurs or if an accident occurs but the individual$ drecides not to claim, then the premium category next period is $u(n)$; alternatively, if an accident occurs and the individual decides to claim, then he or she pays the first $e_{n}$ of the claim (the rest being paid by the company) and next period the premium category is $d(n)$; this process continues indefinitely or terminates randomly. 
All that remains is to determine when the individual should claim. This we do in the next section.

\section{The solution}

It is intuitively obvious that the optimal claiming strategy in any period is of the reservation form: that is, a claim should be made if the accident cost is "sufficiently high"; otherwise a claim should not be made. All that remains to be determined is precisely what is meant by "sufficiently high".

A reservation strategy is characterised by a reservation value (which determines whether a specific value is sufficiently high). Clearly the reservation value depends upon which premium category the individual is in, but it does not depend upon the particular time period under consideration. (Given the premium category, the future always looks the same wherever the individual views it from - because of the assumptions of the model; that is, the model, and its solution, are time-independent. See Hey [1981], pp. $51 \mathrm{ff}$.)

Let us denote an arbitrary reservation value for premium category $n$ by $x_{n}$, and the corresponding optimal reservation value by $x_{n}^{*}$. Thus, an arbitrary (the optimal) reservation strategy when in category $n$ is as follows (where $x$ is the total accident cost in the period):

$$
\left\{\begin{array}{l}
\text { if } x>x_{n}\left(x_{n}^{*}\right) \text { then claim; } \\
\text { if } x \leqslant x_{n}\left(x_{n}^{*}\right) \text { then do not claim. }
\end{array}\right.
$$

The optimal claiming strategy is thus characterised by $x_{1}^{*}, \ldots, x_{N}^{*}$. So all that remains is to determine these. To this end, let us introduce $V_{1}, \ldots, V_{N}$ where $V_{n}$ measures the maximal value of expected lifetime utility (as given by (1)) as viewed from the beginning of a period in which the individual's premium category is $n$. Thus, $V_{n}$ measures the expected value of (1) on the assumption that the individual behaves optimally not only in the current period (in which the individual is in premium category $n$ ) but also in all subsequent periods. being

The $V_{\mathrm{S}}$ are determined implicitly by a set of recursive equations, that for $V_{n}(n=1, \ldots, N)$

$$
\begin{aligned}
V_{n} & =(1-p)\left[U\left(I-P_{n}\right)+\rho V_{u(n)}\right] \\
& +p \max _{x_{n}}\left(\left\{\int_{x=0}^{x_{n}}\left[U\left(I-P_{n}-x\right)+\rho V_{u(n)}\right] d F(x)+\int_{x=x_{n}}^{\infty}\left[U\left(I-P_{n}-e_{n}\right)\right.\right.\right. \\
& \left.\left.\left.+\rho V_{d(n)}\right] d F(x)\right\}\right) .
\end{aligned}
$$

Pershaps a word of explanation is in order. The first term, namely $(1-p)\left[U\left(I-P_{n}\right)+\rho V_{u(n)}\right]$, arises when no accident occurs in the period. This has probability $(1-p)$; and the utility in that period is thus that yielded by "other consumption" of $I-P_{n}$, while the utility over the rest of life is that derived from beginning the following period in premium category $u(n)$. The second term, $p \max (\ldots)$, arises when there is an accident (which has probability $p$ ). The first term in (...) arises when the accident amount $x$ is below the reservation value $x_{n}$; so the individual 
decides not to claim, but to bear the cost him- or herself. Next period the individual is in category $u(n)$ (as if no accident had occurred). The second term in (...) arises when the accident amount $x$ is above the reservation value $x_{n}$; so the individual decides to claim, and thus pays only the first $e_{n}$ of the claim. Next period, however, the individual sinks to category $d(n)$. (Note that (7) implicitly assumes that $x_{n} \geqslant e_{n}$; clearly this must be true for $x_{n}^{*}$, as the reader can easily check.)

The optimal value of $x_{n}$, namely $x_{n}^{*}$, is simply that value at which the maximum of the term on the right-hand-side of (7) is attained. The necessary and sufficient condition (assuming an interior solution ${ }^{1}$ and that $U^{\prime}>0$ ) is

$$
U\left(I-P_{n}-x_{n}^{*}\right)+V_{u(n)}=U\left(I-P_{n}-e_{n}\right)+\rho V_{d(n)} .
$$

(Note that this does not require an assumption of risk-aversion.)

Equation (8) is a familiar type of indifference condition. Its interpretation is straightforward: given an accident cost exactly equal to the optimal reservation claim, then the individual is indifferent between not claiming (which yields the utility on the left-hand side of (8)) and claiming (which yields the utility on the right-hand side of (8)). Thus, it is clear that utility when claiming is greater (less) than utility not claiming when the accident cost is greater (less) than $x_{n}^{*}$.

Before proceeding, it may be useful to note the following (partial) comparative static results which flow from (8) (note that use is made of the obvious fact - see later - that $\left.V_{u(n)}>V_{d(n)}\right)$ :

$$
\begin{aligned}
& \partial x_{n}^{*} / \partial V_{u(n)}>0 \\
& \partial x_{n}^{*} / \partial V_{d(n)}<0 \\
& \partial x_{n}^{*} / \partial e_{n}>0 \\
& \partial x_{n}^{*} / \partial \rho>0 \\
& \partial x_{n}^{*} / \partial I>0 \quad \text { (assuming risk aversion). } \\
& \partial x_{n}^{*} / \partial P_{n}<0 \quad \text { (assuming risk aversion). }
\end{aligned}
$$

Thus, the individual has a higher optimal reservation value (and is thus less likely to claim): the higher is the utility of being in the lower premium category, the lower is the utility of being in the higher premium category; the higher is the deductible; the higher is the rate of time discount; the higher is income; and the lower is the premium payment. Note that neither $p$ nor $F\left(\right.$.) influence $x_{n}^{*}$ (directly), which is intuitively sensible.

However, we must emphasis that the above results are very much partial comparative static results: a full analysis takes into account the fact that changes in $P_{n}, e_{n}, \rho, I, p$ and $F($. change $V_{u(n)}$ and $V_{d(n)}$, and thus affect $x_{n}^{*}$ indirectly as well as directly. As we see later, this renders

\footnotetext{
1 The derivative of the maximand in (7) is always positive at $x_{n}=e_{n}$ (for $\left.V_{u(n)}>V_{d(n)}\right)$; hence $x_{n}^{*}>e_{n}$. If $U$ is unbounded below, then $x_{n}^{*}$ must be finite; though an infinite value is possible if $U$ is bounded below.
} 
most of the complete conparative static effects and ambiguous. (For example, an increase in $p$ does not affect $x_{n}^{*}$ directly, but it does decrease both $V_{u(n)}$ and $V_{d(n)}$; the first of these leads to a fall in $x_{n}^{*}$, the second to a rise in $x_{n}^{*}$. The net effect is, in general, ambiguous.)

Equation (8) determines the value of $x_{n}^{*}$ in terms of $V_{u(n)}$ and $V_{d(n)}$. It therefore remains to find an equation determining $V_{n}(n=1, \ldots, N)$. Clearly if we replace $x_{n}$ on the right-hand-side of (7) by $x_{n}^{*}$ then the maximal value is attained. Thus we get

$$
\begin{aligned}
V_{n} & =(1-p)\left[U\left(I-P_{n}\right)+\rho V_{u(n)}\right] \\
& +p\left\{\int_{x=0}^{x_{n}^{*}}\left[U\left(I-P_{n}-x\right)+\rho V_{u(n)}\right] d F(x)+\int_{x=x_{n}^{*}}^{\infty}\left[U\left(I-P_{n}-e_{n}\right)+\rho V_{d(n)}\right] d F(x)\right\} .
\end{aligned}
$$

This expression can be simplified using integration by parts and the optimality condition (8). This yields ${ }^{2}$

$$
\begin{aligned}
V_{n} & =(1-p)\left[U\left(I-P_{n}\right)+\rho V_{u(n)}\right]+p\left[U\left(I-P_{n}-e_{n}\right)+\rho V_{d(n)}\right] \\
& +p \int_{x=0}^{x_{n}^{*}} U^{\prime}\left(I-P_{n}-x\right) F(x) d x .
\end{aligned}
$$

(As an aside, we note that if the individual always claimed, irrespective of the amount of the accident, then the expression corresponding to (9) would be the same except thet the range of the integration in the final term would be from 0 to $e_{n}$. Thus the immediategain from behaving optimally is

$$
p \int_{x=e_{n}}^{x_{n}^{*}} U^{\prime}\left(I-P_{n}-x\right) F(x) d x
$$

which is necessarily positive since $x_{n}^{*}>e_{n}$ (see earlier). In addition, there would be a further gain since the (sub-optimal) $V_{u(n)}$ and $V_{d(n)}$ would be less than those in (9). End of aside).

Equations (8) and (9) hold, of course, for all $n=1, \ldots, N$. This set constitutes the complete solution of the problem. For convenience we repeat it here:

$$
\begin{aligned}
& U\left(I-P_{n}-x_{n}^{*}\right)+\rho V_{u(n)}=U\left(I-P_{n}-e_{n}\right)+\rho V_{d(n)}, \quad n=1, \ldots, N ; \\
& V_{n}=(1-p)\left[U\left(I-P_{n}\right)+\rho V_{u(n)}\right]+p\left[U\left(I-P_{n}-e_{n}\right)+\rho V_{d(n)}\right] \\
& \quad+p \int_{x=0}^{x_{n}^{*}} U^{\prime}\left(I-P_{n}-x\right) F(x) d x, \quad n=1, \ldots, N .
\end{aligned}
$$

2 The first two terms in (9) are the expected utility if the individual always claims; thus the third term represents the extra utility gained by claiming only when it is optimal so to do. 
Equations (10) consist of $2 N$ equations in the $2 N$ unknowns: $x_{1}^{*}, \ldots, x_{N}^{*}$ and $V_{1}, \ldots, V_{N}$. However, the solution is implicit. An explicit solution cannot, in general, be found because of the nonlinear nature of (10). However, this does not prevent us from calculating numerical solutions in particular instances; nor does it prevent us from exploring the general comparative static implications of the solution. These we do in the next section.

\section{Numerical examples and comparative statics}

This section does two things: first, provides a numerical example; secondly, presents some comparative static propositions.

We begin with a simple, but realistic, numerical example which gives some idea of the relevant orders of magnitude. We need to specify: (1) the individual's utility function, income and discount factor; (2) the distribution of accident costs and the probability of having "an" accident; and (3) the details of the insurance contract.

Under (1), we rather arbitrarily assume in most of our numerical examples that $U$ is of the constant absolute risk aversion form, namely

$$
U(x)=-\exp (-R x)
$$

where $R$ is the (Arrow-Pratt) index of absolute risk aversion. (An increase in $R$ implies an increase in risk aversion.) In Example 1 below we take $R$ to be 0.005 . (This implies that the individual would be indifferent between a gamble which paid $£ x$ or $£(x+100)$, each with probability $\frac{1}{2}$, and a certainty of $\mathfrak{E}(x+43.8)$; so the individual is moderately risk-averse.) We take $\rho$ to be 0.95 and $Y$ to be $£ 5000$.

We put $p$ (the probability of having an accident) equal to 0.06 ; in British terms this represents a relatively safe driver; in contrast, a relatively bad driver has $p$ around 0.14 . We take $F($.), the distribution of total accident costs, to be lognormal with mean $£ 600$ and standard deviation $£ 1800$. (So $\ln x$ is normally distributed with mean 5.2456 and standard deviation 1.5174 .)

We put $N$, the number of premium categories, equal to 5 , and suppose that the $2 \mathrm{nd}, 3 \mathrm{rd}$, 4th and 5th categories represent discounts of $30 \%, 40 \%, 50 \%$ and $60 \%$ on the basic premium (category 1). We take the latter to be $£ 100$. We put the deductible equal to zero in all categories. Finally, we examine two different no claim discount schemes: namely, the "one up/one down scheme" (as characterised in (4)), and the "majority scheme" (as characterised in (5)).

Table 1 presents the values of $x_{1}^{*}, \ldots, x_{s}^{*}$ for the two schemes. These values were obtained by numerical solution of (10). (To be specific, we followed an iterative process: starting with $x_{1}^{*}=\ldots=x_{5}^{*}=0$ we found the implied values of $V_{1}, \ldots, V_{n}$ from $(10 \mathrm{~b})$; these were then substituted into (10a) and new values for $x_{1}^{*}, \ldots, x_{5}^{*}$ found. The iteration continued until convergence was attained; this usually did not require many iterations. We should note that, in all our numerical examples, we verified that $V_{n}>\left\{(1-p) U(I)+p \int_{0}^{\infty} U(I-x) d F(x)\right\} /(1-\rho)$, that is, the
individual was better off insured than uninsured.) 
Table 1: Example 1
(1) $R=.005$
$X=5000$
$\rho=.95$
(2) $p=.06$
$X$ is $A(600,1800)$;

\begin{tabular}{|ccc|cc|}
\hline Category & Premium & Excess & \multicolumn{2}{|c|}{ Reservation value $x_{\mathrm{n}}^{*}$} \\
\cline { 4 - 4 }$n$ & $P_{n}$ & $e_{n}$ & "one up/one down" “majority" \\
\hline 1 & 100 & 0 & 43.75 & 42.96 \\
2 & 70 & 0 & 71.22 & 69.54 \\
3 & 60 & 0 & 44.50 & 86.64 \\
4 & 50 & 0 & 28.83 & 54.91 \\
5 & 40 & 0 & 10.61 & 31.83 \\
\hline
\end{tabular}

Table 1 reveals that with a "one up/one down scheme" the individual should optimally claim only for accidents costing more than $£ 43.75$ when in category 1 , more than $£ 71.22$ when in category 2 , more than $£ 44.50$ when in category 3 , more than $£ 28.83$ in category 4 and more than $£ 10.61$ in category 5. Interestingly, and pershaps counterintuitively, these reservation values reach a peak in category 2 . On reflection this seems reasonable: the individual has more to lose when claiming in category 2 than in category 1 (because there is no punishment from claiming in category 1), and more to lose when claiming in category 2 than in category 3,4 and 5 (because of the non-linearity of the premium scale). The "majority scheme" reservation values, in contrast, reach a peak in category 3 ; this reflects the greater degree of punishment inherent in the "majority scheme". The same factor accounts for the fact that the $x^{*}$ values are generally higher under the "majority scheme" than the "one up/one down scheme".

General comparative static results (concerning the effect on the $x^{*} s$ of changes in the parameters and functions of the problem) are difficult, if not impossible, to obtain. Most are ambiguous, for reasons sketched briefly in section 3. Let us now consider this in more detail.

Comparative static exercises are performed by differentiating totally the solution, as given in (10). Consider, for example, a change in $p$, all other parameters and functions remaining constant. We have, from (10), for $n=1, \ldots, N$ :

$$
-U^{\prime}\left(I-P_{n}-x_{n}^{*}\right) d x_{n}^{*}+\rho d V_{u(n)}-\rho V_{d(n)}=0
$$

and

$$
\begin{aligned}
d V_{n} & -(1-p) \rho d V_{u(n)}-p \rho d V_{d(n)}-p U^{\prime}\left(I-P_{n}-x_{n}^{*}\right) F\left(x_{n}^{*}\right) d x_{n}^{*} \\
& =d p\left\{-\left[U\left(I-P_{n}\right)+\rho V_{u(n)}\right]+\left[U\left(I-P_{n}-e_{n}\right)+\rho V_{d(n)}\right]\right. \\
& \left.+\int_{0}^{x_{n}^{*}} U^{\prime}\left(I-P_{n}-x\right) F(x) d x\right\} .
\end{aligned}
$$


It is clear from (12) that the sign of $\partial x_{n}^{*} / \partial p$ is the same as the sign of $\partial V_{u(n)} / \partial p-\partial V_{d(n)} / \partial p$. To try and find the sign of this we eliminate $x_{n}^{*}$ from (13) using (12) and we use (10b) to simplify the resulting expression. This yields, for $n=1, \ldots, N$ :

$$
\begin{aligned}
d V_{n} & -\rho\left[(1-p)+p F\left(x_{n}^{*}\right)\right] d V_{u(n)}-\rho p\left[1-F\left(x_{n}^{*}\right)\right] d V_{d(n)} \\
& =d p\left[V_{n}-U\left(I-P_{n}\right)-\rho V_{u(n)}\right] / \rho .
\end{aligned}
$$

Clearly (see (10b) and use the obvious fact fact that $V_{u(n)} \geqslant V_{n} \geqslant V_{d(n)}$ ) the coefficient of $d p$ in this is negative. Let us denote this coefficient by $c_{n}$; that is

$$
c_{n} \equiv V_{n}-U\left(I-P_{n}\right)-\rho V_{u(n)}<0 \quad n=1, \ldots, N .
$$

Let us further denote by $a_{n}$ the expression

$$
a_{n} \equiv p\left[1-F\left(x_{n}^{*}\right)\right] \text {. }
$$

Clearly $0<a_{n}<1$.

We can now write (14) as

$$
\partial V_{n} / \partial p-\rho\left(1-a_{n}\right) \partial V_{u(n)} / \partial p-\rho a_{n} \partial V_{d(n)} / \partial p=c_{n}, \quad n=1, \ldots, N .
$$

As we sketch in the Appendix, we can prove from this that

$$
\partial V_{n} / \partial p<0 \quad n=1, \ldots, N,
$$

but we cannot determine whether $\partial V_{u(n)} / \partial p-\partial V_{d(n)} \partial p$ is positive or negative. Thus, see (12) above, the sign of $\partial x_{n}^{*} / \partial p$ is ambiguous.

All we can show in general, therefore, is that an increase in the probability of having "an" accident leads to a decrease in lifetime utility (from wherever viewed). However, the effect on the reservation values is ambiguous. This illustrated in Figure 1, which graphs the $x_{n}^{*}$ as functions of $p$, for values of $p$ from .05 to .95. (The other parameters are the same as in Example 1: Table 1 gives the parametric details.)

It is interesting to note that $x_{n}^{*}$ reaches its peak at a lower value of $p$ than does $x_{n+1}^{*}$ (for $n=$ $1, \ldots, 4)$. This leads to the possibility that the relative positions of $x_{1}^{*}, \ldots, x_{2}^{*}$ change as $p$ changes. Indeed this is the case in Figure 1: in the "one up/one down scheme", at $p=.05$ we have $x_{5}^{*}<x_{4}^{*}<x_{1}^{*}<x_{3}^{*}<x_{2}^{*}$, whereas at $p=.95$ we have $x_{1}^{*}<x_{5}^{*}<x_{2}^{*}<x_{4}^{*}<x_{3}^{*}$. A similar phenomenon occurs with the "majority scheme". It could, of course, be argued that much of Figure 1 is irrelevant, since the "relevant range" (that normally found in the U.K.) is from $p=.06$ to $p=.14$. In this range $\partial x_{n}^{*} / \partial p>0$ for all $n$ in both schemes. Nevertheless, it remains true that the general comparative static effect of a change in $p$ is ambiguous.

The reason is as we have already noted: that $x_{n}^{*}$ depends positively on $V_{u(n)}$ and negatively on $V_{d(n)}$. Thus, in general, any parameter change that alters $V_{u(n)}$ and $V_{d(n)}$ in the same direction has an ambiguous effect on $x_{n}^{*}$. This is not only true of $p$ but also of other parameters. Nevertheless, some further illustrative examples may be of interest. 

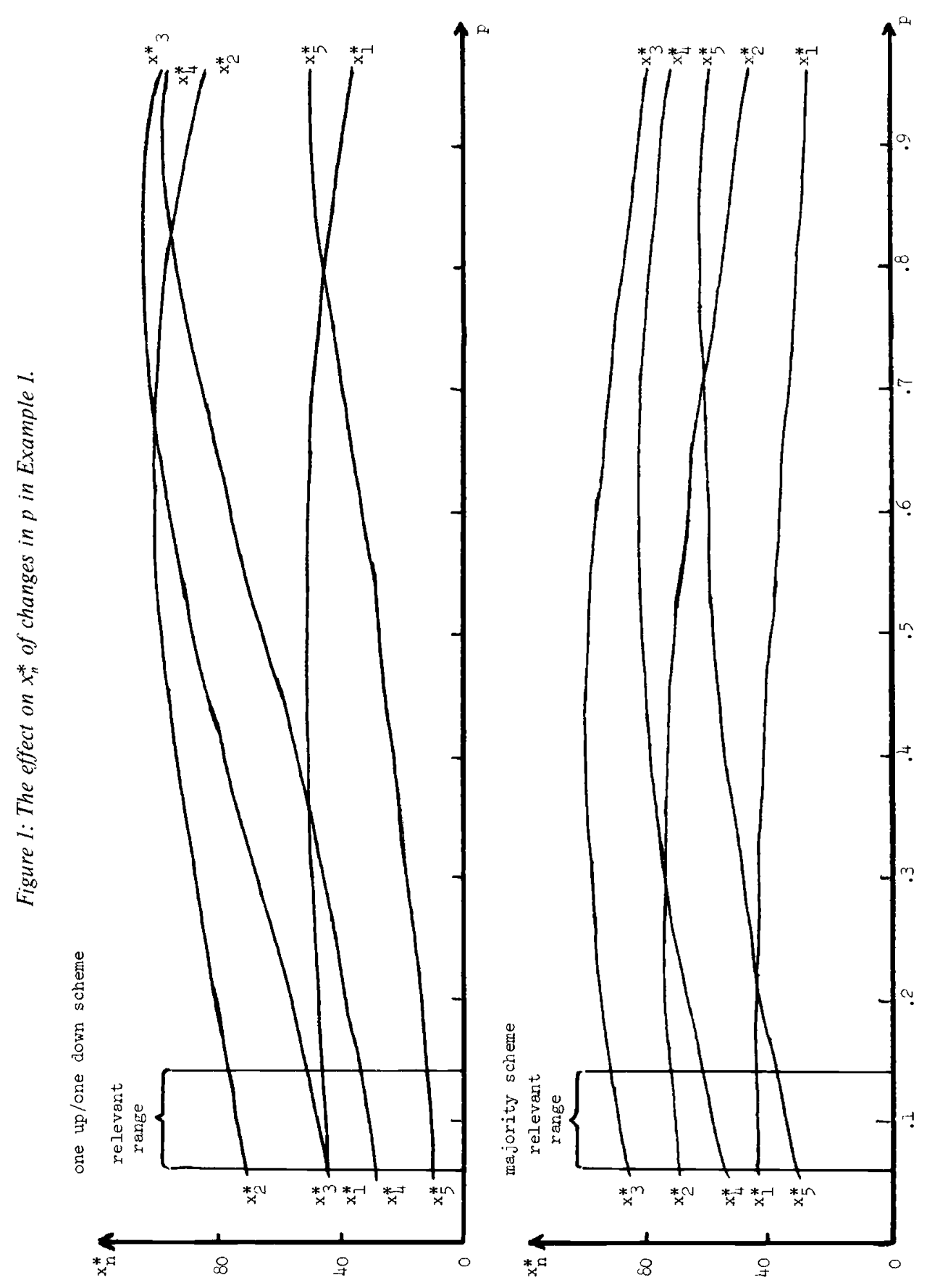
Table 2 illustrates the effect of changes in $\rho$ (the discount factor) on the optimal claim strategy. All other parameters are as in Example 1 (Table 1 gives the parametric details).

Table 2: The effect on $x_{n}^{*}$ of changes in $\rho$ in Example 1

One up/one down scheme

\begin{tabular}{|c|c|r|r|r|r|r|r|r|r|r|}
\hline$\rho$ & .05 & .15 & .25 & .35 & .45 & .55 & .65 & .75 & .85 & .95 \\
\hline$x_{1}^{*}$ & 1.41 & 4.34 & 7.48 & 10.90 & 14.70 & 18.99 & 23.89 & 29.54 & 36.11 & 43.75 \\
$x_{2}^{*}$ & 2.15 & 6.72 & 11.76 & 17.37 & 23.70 & 30.89 & 39.09 & 48.45 & 59.11 & 71.22 \\
$x_{3}^{*}$ & 1.05 & 3.47 & 6.36 & 9.77 & 13.77 & 18.41 & 23.74 & 29.83 & 36.74 & 44.50 \\
$x_{4}^{*}$ & 1.02 & 3.22 & 5.61 & 8.21 & 11.02 & 14.07 & 17.36 & 20.91 & 24.72 & 28.83 \\
$x_{5}^{*}$ & 0.51 & 1.55 & 2.60 & 3.66 & 4.75 & 5.85 & 6.99 & 8.16 & 9.36 & 10.61 \\
\hline
\end{tabular}

Majority scheme

\begin{tabular}{|c|r|r|r|r|r|r|r|r|r|r|}
\hline$\rho$ & .05 & .15 & .25 & .35 & .45 & .55 & .65 & .75 & .85 & .95 \\
\hline$x_{1}^{*}$ & 1.41 & 4.34 & 7.46 & 10.85 & 14.59 & 18.79 & 23.58 & 29.09 & 35.49 & 42.96 \\
$x_{2}^{*}$ & 2.14 & 6.67 & 11.63 & 17.12 & 23.28 & 30.26 & 38.21 & 47.29 & 57.68 & 69.54 \\
$x_{3}^{*}$ & 2.76 & 8.65 & 15.13 & 22.29 & 30.25 & 39.14 & 49.09 & 60.23 & 72.71 & 86.64 \\
$x_{4}^{*}$ & 1.59 & 5.08 & 9.04 & 13.53 & 18.60 & 24.31 & 30.73 & 37.92 & 45.96 & 54.91 \\
$x_{5}^{*}$ & 1.08 & 3.42 & 5.99 & 8.80 & 11.88 & 15.22 & 18.86 & 22.82 & 27.13 & 31.83 \\
\hline
\end{tabular}

A glance at Table 2 reveals that in this instance increases in $\rho$ always lead to increases in $x_{n}^{*}$. Indeed this is what intuition leads us to expect (the more one cares about the future the less likely one is to claim in the present), but it is not a result that appears generally true. However, in all our simulations, $\partial x_{n}^{*} / \partial p$ was al ways positive.

Table 3 illustrates the effect of changes in $R$ (the individual's absolute risk aversion index) on the optimal claim strategy. All other parameters are as in Example 1 (Table 1 gives the details).

Table 3: The effect on $x_{n}^{*}$ of changes in $R$ in Example 1

One up/one down scheme

\begin{tabular}{|c||c|c|c|c|c|}
\hline$R$ & .002 & .004 & .006 & .008 & .010 \\
\hline$x_{1}^{*}$ & 50.48 & 45.80 & 41.88 & 38.54 & 35.67 \\
$x_{2}^{*}$ & 79.51 & 73.72 & 68.95 & 64.93 & 61.50 \\
$x_{3}^{*}$ & 48.14 & 45.64 & 43.44 & 41.48 & 39.74 \\
$x_{4}^{*}$ & 30.13 & 29.25 & 28.43 & 27.67 & 26.96 \\
$x_{5}^{*}$ & 10.59 & 10.60 & 10.61 & 10.62 & 10.63 \\
\hline
\end{tabular}


Majority scheme

\begin{tabular}{|c||l|l|l|l|l|}
\hline$R$ & .002 & .004 & .006 & .008 & .010 \\
\hline$x_{1}^{*}$ & 49.47 & 44.94 & 41.14 & 37.90 & 35.10 \\
$x_{2}^{*}$ & 77.39 & 71.91 & 67.37 & 63.54 & 60.26 \\
$x_{3}^{*}$ & 96.76 & 89.66 & 83.91 & 79.13 & 75.10 \\
$x_{4}^{*}$ & 58.67 & 56.08 & 53.82 & 51.81 & 50.03 \\
$x_{5}^{*}$ & 32.20 & 31.95 & 31.72 & 31.50 & 31.30 \\
\hline
\end{tabular}

Note: a value of $R$ equal to $.002, .004, .006, .008, .010$ means that the individual is different between a 50.50 gamble between $£(100+x)$ and $£ x$ and a certainty of $£(47.50+x), £(42.61+x), £(40.26+x)$, $£(37.99+x)$ respectively.

The general tendency apparent in Table 3 is for an increase in risk aversion to decrease the reservation values $x_{n}^{*}$ : effectively the more risk averse individual is buying more insurance. This makes goods sense. However, there are exceptions to this general rule, as the final row of the "one up/one down" table indicates: here $x_{5}^{*}$ increases as $R$ increases. The intuition behind this result appears obscure.

Within the context of our specific utility function (11), changes in income $I$ clearly have no effect on the $x_{n}^{*}$ - since the utility function (11) displays constant absolute risk aversion at all levels of income. This is confirmed by our numerical examples. With other utility functions, changes in $I$ will clearly have an effect: for example, with a utility function that displays decreasing (increasing) absolute risk aversion, an increase in income will effectively mean that the risk aversion of the individual decreases (increases). The general implication of this (see the paragraph above) will be for the $x_{n}^{*}$ to increase (decrease), though there may be exceptions to this general rule. Thus, in general, people on high incomes are less likely to claim than people on low incomes - if decreasing absolute risk aversion prevails.

Other comparative static exercises are possible: for example, investigating the effect of changes in the distribution of $x$. We have in fact computed a number of illustrative cases; once again, the effects are in general ambiguous. We refrain from reporting them here for reasons of space. However, we would be happy to supply examples to interested readers on request.

\section{The effects of the introduction of a no claim discount scheme}

There are a number of ways that we can assess the impact of the introduction of a no claim discount scheme. One way is to calculate the effect on the individual's level of utility given a specified profit rate for the insurance company. Let us first specify a required gross profit rate - defined as the percentage excess of premium payments over total accident claims paid. A figure of around $30 \%$ seems to be normal in the U.K. If the various parameters are as specified in Example 1, and if the various discounts are as in Example 1, then according to our calculations the required basic premium would be $£ 99.89$ if the company operated a "one up/one down scheme" and $£ 97.37$ if the company operated a "majority scheme". Under the assumption that individuals behave optimally, then the implied gross profit rate is the required $30 \%$. 
Table 4 presents some of the implications of this example. The double-starred ${ }^{* *}$ entries are all per period equivalents, calculated as follows. Let $Y_{n}$ denote the expected lifetime value of the appropriate variable (accident costs, premium payments) as viewed from the beginning of a period in which the policy-holder is in premium category $n$. Then the table entry is $(1-\rho) Y_{n}$ where $\rho$ is the discount factor. For convenience we have used the same discount factor for both the company and the policy-holder (the insuree).

Table 4: Costs and payments yielding gross profit rates of 30\% in Example 1

One up/one down scheme

\begin{tabular}{|c|c||c||c|c|c|}
\hline $\begin{array}{c}\text { Premium } \\
\text { category } \\
n\end{array}$ & $\begin{array}{c}\text { Premium } \\
P_{n}\end{array}$ & $\begin{array}{c}\text { Reservation } \\
\text { value } \\
x_{n}^{*}\end{array}$ & $\begin{array}{c}\text { Accident costs } \\
\text { borne by } \\
\text { insuree }\end{array}$ & $\begin{array}{c}* * \\
\text { Premium } \\
\text { payments by } \\
\text { insuree }\end{array}$ & $\begin{array}{c}* * \\
\text { Accident costs } \\
\text { borne by } \\
\text { insurer }\end{array}$ \\
\hline 1 & 99.89 & 43.71 & 0.07 & 46.71 & 35.93 \\
2 & 69.92 & 71.16 & 0.06 & 43.76 & 35.94 \\
3 & 59.93 & 44.46 & 0.04 & 42.18 & 35.96 \\
4 & 49.94 & 28.80 & 0.02 & 41.11 & 35.98 \\
5 & 39.96 & 10.60 & 0.02 & 40.57 & 35.98 \\
\hline
\end{tabular}

Majority scheme

\begin{tabular}{|c|c||c||c|c|c|}
\hline $\begin{array}{c}\text { Premium } \\
\text { category } \\
n\end{array}$ & $\begin{array}{c}\text { Premium } \\
P_{n}\end{array}$ & $\begin{array}{c}\text { Reservation } \\
\text { value } \\
x_{n}^{*}\end{array}$ & $\begin{array}{c}\text { Accident costs } \\
\text { borne by } \\
\text { insuree }\end{array}$ & $\begin{array}{c}* * \\
\text { Premium } \\
\text { payments by } \\
\text { insuree }\end{array}$ & $\begin{array}{c}* * \\
\text { Accident costs } \\
\text { borne by } \\
\text { insurer }\end{array}$ \\
\hline 1 & 97.38 & 42.08 & 0.22 & 46.52 & 35.78 \\
2 & 68.16 & 68.01 & 0.22 & 43.70 & 35.78 \\
3 & 58.43 & 84.76 & 0.20 & 42.21 & 35.80 \\
4 & 48.69 & 53.65 & 0.17 & 41.14 & 35.83 \\
5 & 38.95 & 31.04 & 0.16 & 40.59 & 35.84 \\
\hline
\end{tabular}

** see text

Note that the sum of accident costs borne by insuree and accident costs borne by insurer always totals 36.00 ; this, of course, is the (per period equivalent of the) expected accident cost (or simply $p E X$, which equals $0.06 \times 600$ in this example). 
One immediate feature of Table 4 is the almost negligible amount of accident costs borne by the insuree - the inplication being, of course, that refraining from claiming (when $x$ $\left.\leqslant x^{*}\right)$ has a relatively negligible effect. Another way of seeing this is to compute the required premium scale under the assumption that the policy-holder behaves sub-optimally and always claims irrespective of the amount of the accident. Under the "one up/one down scheme" this would require a basic premium of $£ 99.74$ to yield a $30 \%$ gross profit rate, and under the "majority scheme" a basic premium of $£ 96.95$ would be required. These are respectively $15 p$ and $43 p$ lower than the requisite premiums if the policy-holder claims optimally. Hardly an earth-shattering difference!

Additionally, one can compute the monetary value of the utility gained by the policy-holder under the existing no claim discount scheme, and compare this with the monetary value of the utility gained if the no claim discount scheme were scrapped and replaced by a constant premium scheme which yielded the same gross profit rate to the insurance company. This we do as follows. Consider an insurance scheme with a constant premium $P$ and a constant excess $e$. Denote the expected lifetime utility under such a policy by $V$. It can be shown that $V$ is given by

$$
V=\left(\frac{1}{1-\rho}\right)\left\{(1-p) U(I-P)+p U(I-P-e)+p \int_{x=0}^{e} U^{\prime}(I-P-x) F(x) d x\right\}
$$

Conversely, any utility can be expressed as a "constant premium equivalent" (for given $e$ ) by the use of equation (19). Thus, we can work out what constant premium would give the individual the same utility as that yielded under any varying premium scale.

Now, under the "one up/one down scheme" discussed above the "constant premium equivalent" for our individual is $£ 47.40$ (assuming zero deductible) as viewed from the beginning of a period in which the individual is in premium category 1 . (For premium categories 2 , 3,4 and 5 the respective "constant premium equivalents" are $£ 44.03, £ 42.30, £ 41.17$ and $£ 40.60$.) The actual per period premium paid (as viewed from the beginning of a period in which the individual is in premium category 1 ) is $£ 46.71$ (seeTable 4). Thus the individual suffers a loss of utility (equivalent to a per period increase in premium of $69 p$ or $1.5 \%$ ) as a direct consequence of the no claims bonus scheme. Clearly the individual, being risk-averse, would prefer a constant premium scheme to a no claim bonus scheme, for a given (expected) per period premium payment. Of course, if the company switched from a no claim bonus scheme to a constant premium scheme, the claim cost would rise : in the example, the per period cost would rise from $£ 35.93$ (see Table 4) to $£ 36$ (that is $p E X$, the expected per period accident cost, since the insuree would no longer meet any of the accident costs). At the same time, the company could increase the (expected) per period premium from $£ 46.71$ to $£ 47.40$ (see above) and still leave the insuree with the same level of utility as under the no claim bonus scheme. Thus the company's gross profit rate would rise from $30 \%(=100(46.71-35.93) / 35.93)$ to $31^{\frac{2}{3}} \%$ $(=100(47.40-36) / 36)$. It is clear, therefore, that in this instance the replacement of a no claim bonus scheme by a constant premium scheme would represent a Pareto optimal improvement: either the expected profit rate could increase with the policy-holder's utility held constant, or the policy-holder's utility could increase with the profit rate held constant (or some 
combination of the two). Furthermore, it is apparent that this is a perfectly general result - it rests simply on the risk-aversion of the policy-holder and the risk-neutrality of the company. ${ }^{3}$

Under the "majority scheme" the (category l) "constant premium equivalent" for our individual is $£ 47.38$. The actual per period premium payment is $£ 46.52$ (see Table 4 ). With the no claim bonus scheme, the costs borne by the insurance company are $£ 35$.78; with a constant premium scheme the cost would be $£ 36$. Thus the company's profit rate could increase from $30 \%$ to $31.6 \%$, without any decrease in the insuree's utility, by switching from a no claim bonus scheme to a constant premium scheme.

It may be of some interest to point out the difference in the transition probabilities (between the various premium categories) and in the stationary probabilities (of the various premium categories) implied by the policy-holder behaving optimally and sub-optimally. Table 5 gives the details. (The parameters are as in Example 1: Table I gives the details.)

\section{Table 5: Transition and stationary probabilities in Example 1}

One up/one down scheme

claiming using optimal rule

always

claiming

\begin{tabular}{|c|c|c|c|c|c|c|c|}
\hline \multirow{3}{*}{$\frac{\begin{array}{c}\text { Premium } \\
\text { category }\end{array}}{1}$} & \multicolumn{5}{|c|}{ Transition probabilities } & \multirow{2}{*}{\multicolumn{2}{|c|}{$\begin{array}{c}\text { Stationary } \\
\text { probabilities }\end{array}$}} \\
\hline & 1 & 2 & 3 & 4 & 5 & & \\
\hline & .05 & .95 & .0 & .0 & .0 & .000008 & .000015 \\
\hline 2 & .04 & .0 & $.9 \dot{5}$ & .0 & .0 & .000170 & .000244 \\
\hline 3 & .0 & .0498 & .0 & .9502 & .0 & .003259 & .003814 \\
\hline 4 & .0 & .0 & .0536 & .0 & .9464 & .057807 & .059756 \\
\hline 5 & .0 & .0 & .0 & .0583 & .9417 & .938756 & .936171 \\
\hline
\end{tabular}

Majority scheme

always

claiming

\begin{tabular}{|c|c|c|c|c|c|c|c|}
\hline \multirow{3}{*}{$\frac{\begin{array}{c}\text { Premium } \\
\text { category }\end{array}}{1}$} & \multicolumn{5}{|c|}{ Transition probabilities } & \multirow{2}{*}{\multicolumn{2}{|c|}{$\begin{array}{c}\text { Stationary } \\
\text { probabilities }\end{array}$}} \\
\hline & \multirow{2}{*}{$\frac{1}{.0502}$} & \multirow{2}{*}{$\begin{array}{l}2 \\
.9498\end{array}$} & \multirow{2}{*}{3} & \multirow{2}{*}{$\begin{array}{l}4 \\
.0\end{array}$} & \multirow{2}{*}{$\begin{array}{l}5 \\
.0\end{array}$} & & \\
\hline & & & & & & .002496 & .004254 \\
\hline 2 & .0448 & .0 & .9552 & .0 & .0 & .004724 & .007344 \\
\hline 3 & .0418 & .0 & .0 & .9582 & .0 & .051610 & .059304 \\
\hline 4 & .0 & .0476 & .0 & .0 & .9524 & .049451 & .055746 \\
\hline 5 & .0 & .0 & .0528 & .0 & .9472 & .891720 & .873352 \\
\hline
\end{tabular}

${ }^{3}$ Though, as a referee points out, we should not glorify this result too much: "if someone is willing to take over risks for no costs he should take all the risks of the world!" 
Table 5 shows, for example, that under the "one up/one down scheme", if the policy-holder is in category 3 some period, then the probability is .0498 that he or she will be in category 2 next period and .9502 that he or she will be in category 4 . Of course, if the individual always claims then the transition probabilities are simply $p(=.06)$ and $1-p(=.94)$ respectively. The difference reflects the occasions when the individual decides not to claim. The stationary probabilities indicate the long-run position - or, equivalently, the distribution of the company's policy-holders. Thus, for example, if all policy-holders behave optimally then $93.8 \%$ of them will be in the lowest premium category (under the "one up/one down scheme") $5.8 \%$ in the next lowest category, and so on. If, on the other hand, all policy-holders always claim then $93.6 \%$ of them will be in the lowest category, $6.0 \%$ in the next lowest and so on. Clearly, as a comparison of the final two columns of Table 5 reveals, the differences are negligible.

We will return to the question of transition and stationary probabilities in a little while, but let us turn for the moment to the issue of claim-processing expenses. It is often argued (see, for example, Bennett [1974] p. 94) that one of the purposes of a no claim bonus scheme is to discourage small claims, on which management expenses are proportionately high. We can investigate this claim by explicitly incorporating claim-processing costs as an additional item of expenditure. Acting on expert professional advice, we choose as our functional form

$$
c p c(x)=a+b x,
$$

where $\mathfrak{f} c p c(x)$ is the claim-processing-cost for a claim of $\mathfrak{E} x$. In the numerical examples that follow, we put a equal to 12 and $b$ equal to 0.06 . Clearly the average cost of processing claims is a decreasing function of claim size, and for very small claims the average cost is very high.

If these claim-processing-costs are incorporated into Example 1, total costs become $£ 38.76$ per period in the "one up/one down scheme" and $£ 38.53$ in the "majority scheme". These represent additions of $£ 2.83$ and $£ 2.76$ respectively - slightly less than the average claim processing cost on all accidents $(=p(a+b E X)=.06(12+.06 \times 600)=£ 2.88)$ because some of the accidents are paid for by the policy-holder, so no claim-processing cost is incurred. The net profit rates now become $20.64 \%$ and $23.96 \%$ respectively (as compared with gross profit rates of $30.15 \%$ and $33.51 \%$ respectively). However, the interesting comparison is that between the net profit rates if the policy-holder acts optimally and the net profit rates if the policy-holder always claims, irrespective of the accident amount. The latter rates are $20.68 \%$ and $24.15 \%$ respectively. Paradoxically these are greater than the net profit rates under the policy-holder's optimal strategy! Thus, contrary to the assertion above, a no claim bonus scheme can actually decrease net profit rates!

(The explanation is not hard to find: for example, under the "one up/one down scheme" premium payments are $£ 46.76$ per period if the individual behaves optimally and $£ 46.92$ per period if the individual always claims - a difference of $16 \mathrm{p}$ per period; the corresponding total costs borne by the insurer figures are $£ 38.76$ and $£ 38.88$ respectively - a difference of $12 p$ per period. Thus revenue goes up by more than costs when the insuree behaves sub-optimally; hence the increased profit rate. Of course, a necessary condition for this phenomenon is that the insuree is risk-averse.)

Our analysis so far has revealed that a no claim bonus scheme will decrease the policyholder's utility, and may decrease the insurance company's net profit rate. These results hardly provide strong justification for the use of such schemes. However, our analysis has so far ignored possibly the major reason for the existence of such schemes: the desire "to charge 
premiums more closely reflecting the individual risks" Bennett [1974] p. 95). To investigate whether a no claim bonus scheme succeeds in this respect, we present in Table 6 an analysis of stationary probabilities, total costs, revenues and profit rates for different values of $p$, the probability of having "an" accident. We consider values over the U.K. "relevant range" - from .06 to .14. The parameters used in Table 6 are those of example 1 (details in Table 1), while processing-costs (as given by (20) with $a=12$ and $b=.06$ ) are included in total costs.

Table 6: The effect of different $p$ on probabilities, revenues, costs and profits in Example 1 (assuming optimal behaviour)

One up/one down scheme

\begin{tabular}{|ll|c|c|c|}
\cline { 2 - 4 } \multicolumn{1}{c|}{} & $p=.06$ & $p=.10$ & $p=.14$ \\
\hline Stationary & 1 & .000008 & .000064 & .000259 \\
probabilities & 2 & .000170 & .000807 & .002256 \\
(by premium & 3 & .003259 & .009144 & .017988 \\
category) & 4 & .057807 & .095010 & .130500 \\
& 5 & .938757 & .894975 & .848997 \\
\hline Premium payments** & & 46.76 & 47.51 & 48.33 \\
Total costs (insurer)** & & 38.76 & 64.56 & 90.32 \\
Profit rate & $20.64 \%$ & $-26.41 \%$ & $-46.48 \%$ \\
\hline
\end{tabular}

Majority scheme

\begin{tabular}{|c|c|c|c|c|}
\hline & & $p=.06$ & $p=.10$ & $p=.14$ \\
\hline Stationary & 1 & .002496 & .007271 & .014869 \\
\hline probabilities & 2 & .004724 & .012687 & .023960 \\
\hline (by premium & 3 & .051610 & .082902 & .111253 \\
\hline \multirow[t]{2}{*}{ category) } & 4 & .049451 & .077181 & .100600 \\
\hline & 5 & .891720 & .819959 & .749318 \\
\hline Premium payments** & & 47.76 & 49.25 & 50.87 \\
\hline Total costs (insurer) ${ }^{* *}$ & & 38.53 & 64.10 & 89.59 \\
\hline Profit rate & & $23.96 \%$ & $-23.16 \%$ & $-43.22 \%$ \\
\hline
\end{tabular}

** per period rates (see explanation to Table 4).

It is clear from Table 6 that an increase in $p$ implies that the individual spends more time in the high premium categories and less time in the low premium categories. Consequently, the average premium paid increases with $p$. So there is clearly a positive relationship between average premium paid and the individual's risk. Unfortunately, the relationship is not 
particularly strong: for example, under the "one up/one down scheme" the average premium goes up by just $3.4 \%$ while $p$ goes up by $133 \%$; at the same time total claim costs rise by $133 \%$. So the profit rate falls from $+20.64 \%$ to $-46.48 \%$. Things are slightly better under the "majority scheme": the premium goes up by $6.5 \%$ rather than by $3.4 \%$. Nevertheless, this is woefully insufficient to compensate for the $133 \%$ rise in costs (and $p$ ); hemce the profit rate falls from + $23.96 \%$ to $-43.22 \%$. The clear message to emerge from this analysis is that the scheme needs to be much more punitive than either of these for it to have any significant effect. Bur even so, there is a deeper underlying problem - with $p$ as low as (say) . 10 one needs as awful lot of observations before one can estimate it with a reasonable degree of accuracy - which is, of course, what a no claim bonus scheme is trying to do if it purports to exist for the purpose of charging "premiums more closely reflecting the individual risks". Suffice it to say that with the empirically-relevant values for $p$, and with the type of no claim bonus schemes currently in use, there is virtually no chance that the scheme will be successful in this respect.

\section{Summary and conclusions}

The first purpose of this paper was to set up an insurance claim decision problem, and to find its optimal solution. Our formulation, we believe, is reasonably realistic, and contains the main features present in the real world - most notably the dynamic aspects of real-life problems, and the risk aversion displayed by purchasers of insurance. We showed that the optimal claim strategy is characterised by a set of reservation values - one for each premium category. We derived the (implicit) equations determining these reservation values, and calculated their specific values in a number of empirically-relevant examples. (We would be happy to provide solutions to other examples, or indeed to provide the computer program which calculates the solution, to interested readers.) We also showed that, in general, unambiguous comparative static propositions concerning the effect of parameter changes on these reservation values were unobtainable.

The second purpose of the paper was to explore the implications for both the policy-holder and the insurance company of the use of a no claim bonus scheme. We showed that the policy-holders were made worse off as a consequence (because of their aversion to risk), and that the net profit of the insurance company could fall (for parameters in the empiricallyrelevant range). We found little evidence to support the notion that no claim bonus schemes could significantly reduce processing costs through the discouragement of small (expensiveto-process) claims.

We did find that no claim bonus schemes imply a (positive) relationship between accident probabilities and premium payments. However, the relationship is very weak (for parameters in the economically relevant range), and clearly insufficient to compensate for the strong relationship between accident probabilities and accident costs.

Thus overall there seems very little justification for the use of such schemes.

However, our model does exclude two possible sources of justification, in that we assume away: (1) any relationship between the accident probability and the contractual arrangements of the insurance scheme; and (2) any relationship between the accident probability and the previous accident experience. As far as (1) is concerned, Bennett ([1974] p. 95) notes

"It could be argued that the existence of a financial penalty on claiming may induce safer driving but there can be no evidence to support this and is seems unlikely that the amount of potential penalty could have any significant effect." 
So perhaps we can dismiss this. However, Bennett sees more truth in (2). Indeed he concludes his paper with the words:

"If - as in private car insurance in the U.K. - there is evidence that policy-holders' future claims experience varies appreciably ... according to whether or not they have previously made claims, then ... insurers must take this into account in the premiums they charge. Thus ... some form of experience rating such as N.C.D. is inevitable. This, fundamentally, is the answer to the question 'Why N.C.D.?'”

In the context ot our model, this would imply that $p$ (but not the premium directly) would depend upon the individual's accident record. This would appear to be the obvious next step in our research. In the meantine, this paper has confirmed Bennett's professional intuition to the effect that, if $p$ is unrelated to the individual's accident record, there is little justification for the use of no claim bonus schemes.

\section{Appendix}

(1) Sketch of proof of (18)

For illustrative purposes, consider the "one up/one down scheme". The proof for other schemes is similar.

Assume on the contrary that some $\partial V_{n} / \partial p$ is positive. Take $n$ as the lowest value such that $\partial V_{n} / \partial p>0$. Thus $\partial V_{i} / \partial p<0$ for $i=1, \ldots, n-1$.

If $n=1$. We have, from (17), that

$$
\partial V_{2} / \partial p=\left[\left(1-\rho a_{1}\right) / \rho\left(1-a_{1}\right)\right]\left(\partial V_{1} / \partial p\right)-c_{1} /\left[\rho\left(1-a_{1}\right)\right]
$$

Hence if $\partial V_{1} / \partial p>0$, it follows that $\partial V_{2} / \partial p>\partial V_{1} / \partial p>0$.

If $1<n<N$. Either $\partial V_{n} / \partial p>0$ and $\partial V_{n-1} / \partial p<0$, or both are positive, in which case $\partial V_{n} / \partial p>\partial V_{n-1} / \partial p$ by the above argument. Clearly therefore in either case $\partial V_{n} / \partial p>\partial V_{n-1} / \partial p$.

Now we have from (17) that

$$
\begin{aligned}
\partial V_{n+1} / \partial p & =\left(\partial V_{n} / \partial p-p a_{n} \partial V_{n-1} / \partial p-c_{n}\right) /\left[\rho\left(1-a_{n}\right)\right] \\
& >\left(\partial V_{n} / \partial p\right)\left[\left(1-\rho a_{n}\right)\right]>\left(\partial V_{n} / \partial p\right) .
\end{aligned}
$$

Hence the induction continues.

Now consider $n=N$. We have, from (17), that

$$
\left[1-\rho\left(1-a_{N}\right)\right]\left(\partial V_{N} / \partial p\right)=\rho a_{N} \partial V_{N-1} / \partial p+c_{N} .
$$


Now the left-hand-side of this is greater than $\rho a_{n}\left(\partial V_{N} / \partial p\right)$ which is greater than $\rho a_{N}\left(\partial V_{N-1} / \partial p\right)$. But the right-hand-side is less than $\rho a_{N}\left(\partial V_{N-1} / \partial p\right)$. Hence we have a contradiction.

Thus our original supposition must have been wrong. Thus all the $\partial V_{n} / \partial p$ are negative.

QED.

\section{REFERENCES}

BENNETT, M. C. [1974]: "Why N.C.D.?", Journal of the Institute of Actuaries Students' Society, 21 (October 1974), 87-110.

HEY, J. D. [1981]: Economics in Disequilibrium, Martin Robertson, Oxford.

LEMAIRE, J. [1976]: "Driver versus company", Scandinavian Actuarial Journal, 209-219.

LEMAIRE, J. [1977]: "La soif du bonus", A.S.T.I.N. Bulletin, 9, 181-190.

VENEZIA, I. and LEVY, H. [1980]: "Optimal claims in automobile insurance", Review of Economic Studies, 47 (April 1980), 539-549.

ZWEIFEL, P. and GNAEDINGER, A. [1983]: "Bonus systems in insurance: The case of automobile insurance", Paper presented at the 10th Seminar of the European Group of Risk and Insurance Economists, Rome, September 1983. 